\title{
Componentes y actividades del diagnóstico de salud poblacional
}

\author{
Components and activities of the population health diagnosis
}

\section{Ernesto Cotonieto-Martínez $z^{a}$}

\begin{abstract}
:
The health diagnosis is a tool that allows to know the needs and strengths of the health-disease process in a specific population and have the purpose of contributes to health situation through the integration of three diagnoses: administrative, strategic and ideological. It's a continuous process that includes health problem definition, collection of information, interpretation of information and proposals for intervention. This document summarizes the components and activities involved in that process and are represented graphically.
\end{abstract}

Keywords:

Diagnosis of Health Situation, Prioritization of problems, Community Health Planning, Public Health.

\section{Resumen:}

El diagnóstico de salud es una herramienta que permite conocer las necesidades y fortalezas sobre el proceso de salud-enfermedad en una población específica y tiene como objetivo el poder contribuir a la situación de salud a través de la integración de tres diagnósticos: administrativo, estratégico e ideológico. Es un proceso continuo que incluye: definición del problema de salud, recolección de información, interpretación de información y propuestas de intervención. En este documento se resumen los componentes y actividades involucrados en dicho proceso y se representan gráficamente.

\section{Palabras Clave:}

Diagnóstico de la Situación de Salud, Priorización de problemas, Planificación en Salud Comunitaria, Salud Pública.

\section{Introducción}

El diagnóstico de salud es un proceso investigativo a través del cual se identificar las necesidades y problemas, las áreas de fortaleza, así como el papel de los determinantes sociales que inciden en el proceso saludenfermedad y los recursos y servicios disponibles. ${ }^{1}$

Este proceso tiene como meta el poder construir las bases para el diseño e implementación de propuestas de intervención viables y congruentes con la realidad ${ }^{1}$. Por lo que, es imperativo que se lleve a cabo bajo los principios del método científico, utilizando herramientas del enfoque tanto cualitativo como cuantitativo, según las necesidades. En términos generales, existen tres enfoques en los que se pueden clasificar los diagnósticos de salud. ${ }^{1-2}$
Administrativo. Sirve para dar cuenta sobre cómo están funcionando los servicios sanitarios públicos, por lo que suelen utilizarse indicadores epidemiológicos, disponibilidad de insumos y recursos, así como la calidad de la atención.

Estratégico. Sirve para establecer la relación entre las diversas variables que están jugando un papel respecto al proceso de salud-enfermedad, de tal forma que permita identificar factores de riesgo y de protección ante enfermedades, incluyendo los determinantes de la salud.

Ideológico. Buscan acercarse a conocer la realidad de los individuos en cuanto al acceso a los servicios de atención en salud, los aspectos y socioculturales que lo impiden o facilitan.

a Autor de Correspondencia. Universidad Autónoma del Estado de Hidalgo. Instituto de Ciencias de la Salud. ORCID: https://orcid.org/00000001-5639-5898, Email: cotonietoe@gmail.com 


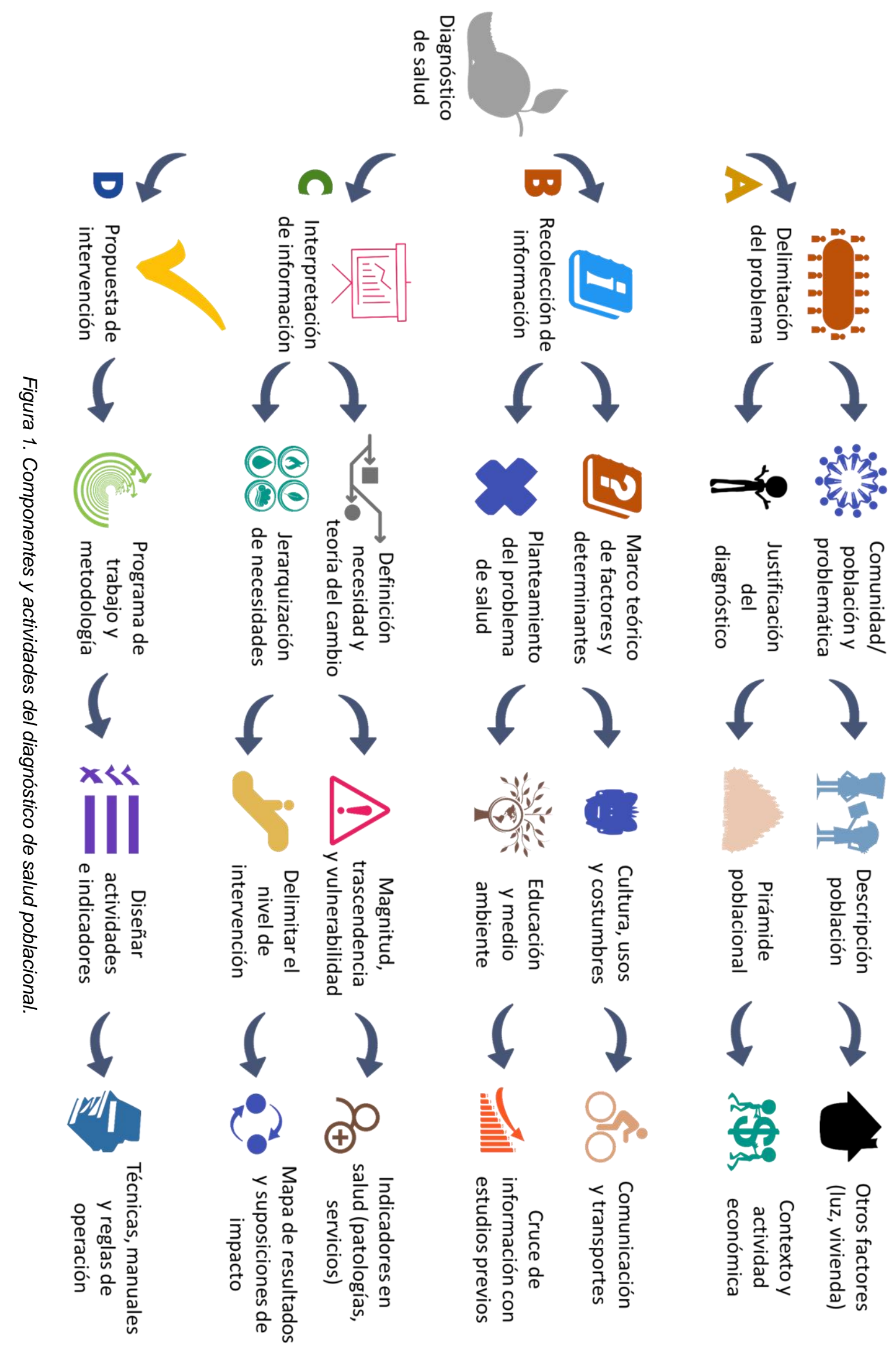


Un diagnóstico de salud integral es aquel capaz de integrar e interpretar información lo suficientemente amplia, pero a la vez estrictamente necesaria que permita tener un panorama claro sobre el proceso saludenfermedad en una población específica. ${ }^{3}$ Lo que incluye los siguientes componentes y actividades (Figura 1):

a) Delimitación del problema. Se describe el diagnóstico a realizar, quienes estarán participando (reseña curricular) así como la importancia y justificación del porqué llevar a cabo el diagnóstico en esa población. Por otro lado, se hace una revisión sociohistórica de la comunidad, ubicación geográfica y localización de referencia, tasa de crecimiento (en los últimos 5 años) se describe la población a través de una pirámide poblacional para conocer la distribución.

b) Recolección de información. Se hace una revisión las características socio-culturales (usos y costumbres); acceso a servicios de educación, nivel educativo, analfabetismo; condiciones ambientales como indicadores de contaminación (y fuentes), flora y fauna, tipo de suelo, clima, etc.; de los indicadores como tasas (natalidad, fertilidad, desarrollo humano, principales morbilidades, etc.), saneamiento básico (agua potable, drenaje, manejo de basura, etc.), recursos y servicios (equipo de profesionales, unidades de atención, asignación de recursos financieros y materiales, cobertura, calidad); caracterización de los medios de comunicación y transporte disponibles; factores socioeconómicos como (actividades económicas, personas económicamente activas, empleo formal, salario e ingresos, movilidad migratoria, Producto Interno Bruto); otros factores como la vivienda (materiales, número de habitantes por vivienda, servicios), luz, actividades recreativas y de esparcimiento.

c) Interpretación de información. Con la información anterior es posible realizar un diagnóstico de salud de una comunidad y población desde un enfoque ecológico; lo que permite identificar y jerarquizar las necesidades de atención a través de la descripción de la magnitud, trascendencia y vulnerabilidad respecto a los problemas de salud encontrados, se delimita e nivel y área de intervención. Posteriormente, se debe revisar la información científica disponible para establecer relaciones causales a través de una teoría del cambio (estudios previos, políticas similares exitosas, consulta de expertos, teorías explicativas). Finalmente, se formulan mediante un mapa de resultados a alcanzar (congruentes con el nivel de atención, los recursos disponibles y la teoría del cambio) y se consideran los factores que pudiesen afectar negativamente en el logro de las metas. d) Propuesta de intervención. Para finalizar el proceso de diagnóstico, se tiene que formular un programa de trabajo o de intervención, así como la metodología utilizada para el diseño, seguimiento y evaluación de impacto de las acciones implementadas. Lo que en primera instancia implica diseñar las actividades, establecer las técnicas, elaborar manuales, definir las reglas de operación y los indicadores estratégicos y de desempeño.

\section{Consideraciones finales}

Si bien es cierto que el diagnóstico podría darse por finalizado con la interpretación de la información, en realidad valdría la pena preguntarse ¿Qué sentido tuvo entonces realizar el diagnóstico?, y seguramente la respuesta más apropiada sería: No tuvo ningún sentido.

Conocer la situación de salud de una población no es la meta, o no debería serlo ni siquiera en el ámbito de la investigación. Este ejercicio adquiere sentido cuando encamina propuestas factibles de intervención que permitan solucionar las problemáticas y necesidades identificadas y potenciar los recursos y fortalezas de una población para poder contribuir a mejores condiciones de salud.

\section{Referencias}

[1] Arenas-Monreal, L., Cortez-Lugo, M., Parada-Toro, I., PachecoMagaña, L. \& Magaña-Valladares. (2015). Diagnóstico de salud poblacional con enfoque de ecosalud. Revista de Saúde Pública, 49(78), $1-8$.

[2] Cordera, A., Kuthy, J., Sucilla, H. (1986). Diagnóstico situacional en sistemas de atención médica. Salud Pública Méx., 28, 243-249.

[3] Mendoza M. Salud Pública I: Productos del Módulo. (Generación 20052007) [Internet]. 2007. México: Universidad Veracruzana. Disponible en: https://www.uv.mx/msp/files/2013/01/dx-2005.pdf 